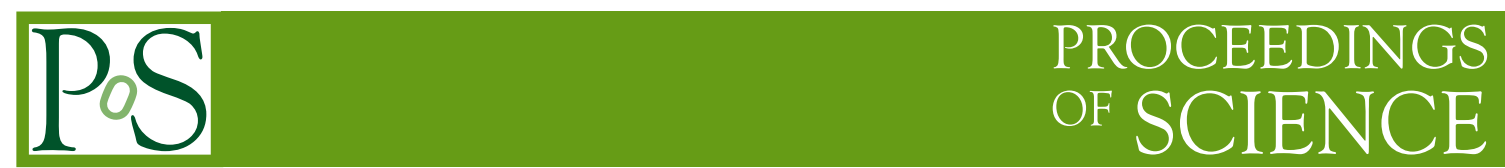

\title{
Lectures on Statistics and Machine Learning
}

\section{Daniel Whiteson*}

Dept. of Physics and Astronomy, University of California, Irvine, CA 92627

E-mail: danieleuci.edu

These lectures covered the basics of statistics and machine learning for collider physics.

Theoretical Advanced Study Institute Summer School 2018 'Theory in an Era of Data'(TASI2018)

4 - 29 June, 2018

Boulder, Colorado

${ }^{*}$ Speaker. 


\section{Statistics}

My statistics lectures were based on Ref [1].

\section{Machine Learning}

My machine learning lectures were based on Ref [2].

\section{References}

[1] K. Cranmer, doi:10.5170/CERN-2015-001.247, 10.5170/CERN-2014-003.267 arXiv:1503.07622 [physics.data-an].

[2] D. Guest, K. Cranmer and D. Whiteson, Ann. Rev. Nucl. Part. Sci. 68, 161 (2018) doi:10.1146/annurev-nucl-101917-021019 [arXiv:1806.11484 [hep-ex]]. 